\title{
Diagonally implicit block backward differentiation formula for solving linear second order ordinary differential equations
}

\begin{abstract}
The three point block method for solving second order ordinary differential equations (ODEs) directly using constant step size is derived. The reliability of this new method is verified in the numerical results with the improved performance in terms of computation time while maintaining the accuracy. The comparison is presented between the new method and classical backward differentiation formulas (BDF) of order 3.
\end{abstract}

Keyword: Block method; Second order ordinary differential equations (ODEs); Backward differentiation formula 